\title{
Chiral recognition ability of cellulose derivatives bearing pyridyl and bipyridyl residues as chiral stationary phases for high-performance liquid chromatography
}

\author{
Yasunaka Katoh $^{1}$, Yasutaka Tsujimoto ${ }^{1}$, Chiyo Yamamoto $^{2}$, Tomoyuki Ikai ${ }^{3}$, Masami Kamigaito ${ }^{1}$ \\ and Yoshio Okamoto ${ }^{3,4}$
}

Cellulose derivatives bearing pyridyl and bipyridyl residues were synthesized, and their recognition abilities as chiral stationary phases for high-performance liquid chromatography were evaluated. Compared with cellulose derivatives bearing these residues at the 2-, 3- and 6-positions of a glucose ring, the regioselectively substituted derivatives exhibited relatively high chiral recognition. The recognition ability of the derivatives was significantly influenced by the coordination of a $\mathrm{Cu}(\mathrm{II})$ ion to the bipyridyl residues. In addition, the derivatives were also used for ligand-exchange chromatography with an eluent containing a copper salt to directly separate amino acids without derivatization.

Polymer Journal (2011) 43, 84-90; doi:10.1038/pj.2010.108; published online 17 November 2010

Keywords: chiral separation; chiralcel; HPLC; ligand exchange; resolution

\section{INTRODUCTION}

Chirality is an attractive property in many scientific fields, including pharmaceuticals, agrochemicals, fragrances, foods, ferroelectric liquid crystals and organic nonlinear optical molecules. Particularly, obtaining both enantiomers with high optical purity is indispensable during the development of chiral drugs, and the use of a single enantiomer is frequently required in the pharmaceutical industry. ${ }^{1-8}$ The chiral separation of racemic compounds is a potential way to obtain pure optically active compounds. ${ }^{9}$

During the past three decades, direct enantioseparation using chiral stationary phases (CSPs) by high-performance liquid chromatography (HPLC) has advanced significantly as a practical method suitable for both analytical and preparative purposes. ${ }^{10-18}$ Chiral separation by HPLC is based on the different interaction behavior between two enantiomers on the CSPs.

Among the large number of CSPs for HPLC derived from chiral small molecules and polymers with chiral recognition abilities, the benzoates and phenylcarbamates of polysaccharides, including cellulose and amylose, have been recognized as the most powerful CSPs for the resolution of a wide range of racemates. ${ }^{19-25}$ However, not all chiral compounds can be resolved using the present polysaccharidebased CSPs. Therefore, a critical challenge to the development of novel CSPs based on polysaccharides must still be met.
In this study, cellulose derivatives bearing pyridyl $\mathbf{1}$ and bipyridyl 2-6 residues (Figures 1 and 2) were synthesized, and their recognition abilities as CSPs for HPLC were evaluated and compared with the commercially available cellulose-based CSPs. The ability to change through a coordination of $\mathrm{Cu}$ (II) ion to the bipyridyl groups of the derivatives 2-6 was also investigated. In addition, the derivatives bearing bipyridyl residues were applied to the resolution of amino acids in ligand-exchange chromatography.

\section{EXPERIMENTAL PROCEDURE}

Materials

Cellulose (Avicel, DP 200) and ammonium acetate were purchased from Merck (Darmstadt, Germany). Methacrolein, formamide, pyridinium tribromide and copper(II) triflate were obtained from Aldrich (Milwaukee, WI, USA). Lithium chloride, 2-acetylpyridine, hydrobromic acid, 2-pyridinecarboxylic acid, 4-pyridinecarboxylic acid and thionyl chloride were purchased from Wako (Osaka, Japan). Benzoyl chloride, triphenylmethyl chloride, potassium permanganate, dichloromethane, 3-pyridinecarboxylic acid, acetic acid and copper(II) sulfate were from Kishida (Osaka, Japan). Triethylamine, 6-chloro3-pyridinecarboxylic acid and oxalyl chloride were from Tokyo Kasei (Tokyo, Japan). Chiralcel OD $(25 \times 0.46 \mathrm{~cm}$ ID), Chiralcel OB $(25 \times 0.46 \mathrm{~cm} \mathrm{ID)}$ and $3,5-$ dimethylphenyl isocyanate were kindly supplied by Daicel Chemical Industries (Tokyo, Japan). Macroporous spherical silica gel (Daiso gel SP-1000) with a mean particle size of $7 \mu \mathrm{m}$ and a mean pore diameter of $100 \mathrm{~nm}$ was kindly

${ }^{1}$ Department of Applied Chemistry, Graduate School of Engineering, Nagoya University, Furo-cho, Chikusa-ku, Nagoya, Japan; ${ }^{2}$ Suzuka National College of Technology, Shiroko-cho, Suzuka, Japan; ${ }^{3}$ EcoTopia Science Institute, Nagoya University, Furo-cho, Chikusa-ku, Nagoya, Japan and ${ }^{4}$ College of Material Science and Chemical Engineering, Harbin Engineering University, Nantong St Harbin, PR China

Correspondence: Professor Y Okamoto, Nagoya University, Furo-cho, Chikusa-ku, Nagoya 464-8603, Japan.

E-mail: okamoto@apchem.nagoya-u.ac.jp

Received 21 August 2010; revised 22 September 2010; accepted 23 September 2010; published online 17 November 2010 

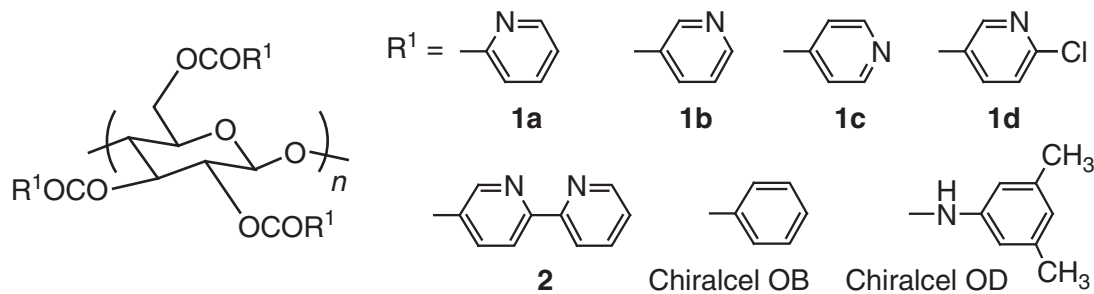

Figure 1 Structures of cellulose derivatives bearing pyridyl groups 1, bipyridyl group 2, Chiralcel OB and Chiralcel OD.
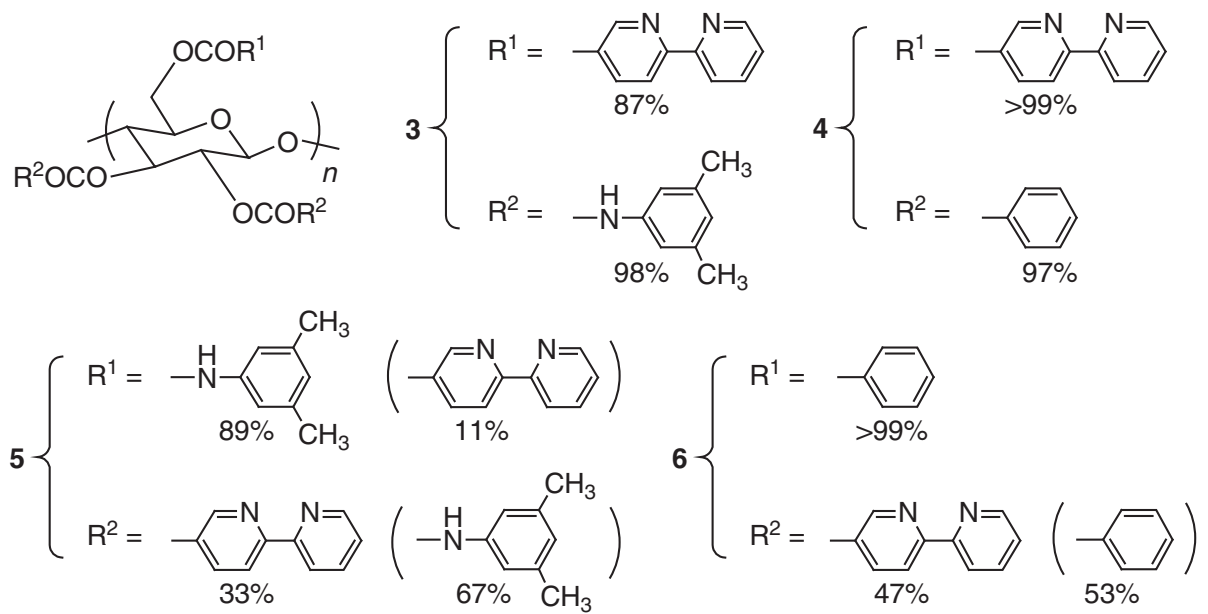

Figure 2 Structures of regioselectively substituted cellulose derivatives bearing bipyridyl group 3-6.

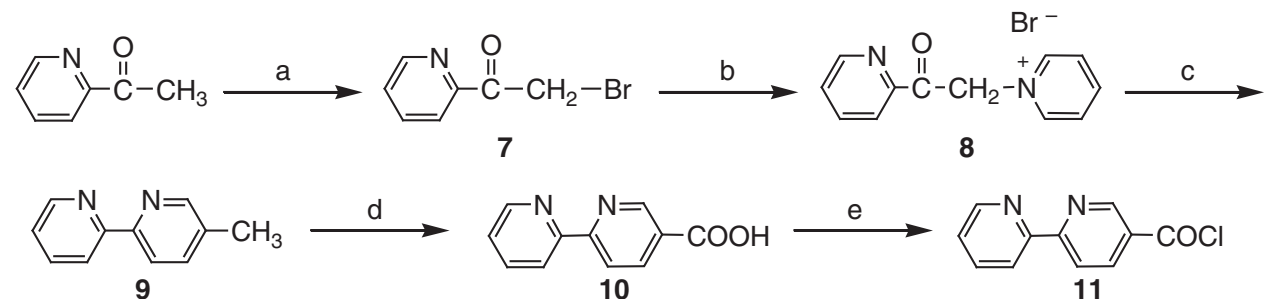

Figure 3 Synthesis of 2,2'-bipyridine-5-carboxylic acid chloride 11. (a) Pyridinium tribromide, $\mathrm{HBr}, \mathrm{AcOH}, 60^{\circ} \mathrm{C}$ for $6 \mathrm{~h}$; (b) pyridine, tetrahydrofuran, $25^{\circ} \mathrm{C}$ for $6 \mathrm{~h}$; (c) methacrolein, $\mathrm{NH}_{4} \mathrm{OAc}$, formamide, $70^{\circ} \mathrm{C}$ for $6 \mathrm{~h}$; (d) $\mathrm{KMnO}_{4}, \mathrm{H}_{2} \mathrm{O}, 70^{\circ} \mathrm{C}$ for $3 \mathrm{~h}, 90^{\circ} \mathrm{C}$ for $12 \mathrm{~h}$; and (e) $\mathrm{SOCl}_{2}, 70^{\circ} \mathrm{C}$ for $3 \mathrm{~h}$.

supplied by Daiso Chemical (Tokyo, Japan). Pyridine, $N, N$-dimethylacetamide, toluene and tetrahydrofuran were purchased from Kanto (Tokyo, Japan) as anhydrous solvents. The racemates were commercially available or were prepared by the usual method. ${ }^{26}$

\section{Synthesis of 2-(bromoacetyl)pyridine (7)}

Following established procedures, ${ }^{27,28}$ a solution of 2-acetylpyridine $(10 \mathrm{~g})$ in acetic acid $(21 \mathrm{ml})$ containing $32 \%$ hydrobromic acid was cooled to $0{ }^{\circ} \mathrm{C}$, and a solution of pyridinium tribromide $(25 \mathrm{~g})$ in acetic acid $(300 \mathrm{ml})$ was added and allowed to react at $60^{\circ} \mathrm{C}$ for $6 \mathrm{~h}$ (Figure 3). The reaction system was cooled to room temperature, and diethyl ether $(600 \mathrm{ml})$ was added to the system. After $12 \mathrm{~h}$ at $0{ }^{\circ} \mathrm{C}$, the precipitates were filtered, washed with acetone and vacuumdried to yield 7 as a yellow solid ( $21 \mathrm{~g},>99 \%)$. The product 7 was used in the next step without further purification.

Synthesis of $\mathrm{N}$-(2-pyridylcarbonylmethyl)pyridinium bromide (8) Pyridine $(10 \mathrm{ml})$ was added to a dispersion of compound $7(20 \mathrm{~g})$ in tetrahydrofuran $(350 \mathrm{ml})$ under a nitrogen atmosphere, and the reaction mixture was stirred at $25^{\circ} \mathrm{C}$ for $6 \mathrm{~h}^{29}$ The precipitate was filtered, washed with tetrahydrofuran and vacuum-dried to give 8 as a white solid (23 g, 82\%). The obtained 8 was used for the preparation of compound 9 without further purification.

\section{Synthesis of 5-methyl-2,2'-bipyridine (9)}

Under a nitrogen atmosphere, methacrolein $(8 \mathrm{~g})$ was added to formamide $(80 \mathrm{ml})$ containing compound $8(23 \mathrm{~g})$ and ammonium acetate $(14 \mathrm{~g})$, and the reaction mixture was stirred at $70^{\circ} \mathrm{C}$ for $6 \mathrm{~h}^{30}$ The precipitate was extracted with diethyl ether and washed with $\mathrm{NaCl}$ aqueous solution. The organic layer was dried over anhydrous $\mathrm{MgSO}_{4}$ and evaporated in vacuo to give product 9 as a yellow oil $(5 \mathrm{~g}, 36 \%)$.

\section{Synthesis of 2,2'-bipyridine-5-carboxylic acid (10)}

The compound $9(8 \mathrm{~g})$ was dispersed in water $(18 \mathrm{ml})$, and $\mathrm{KMnO}_{4}(35 \mathrm{~g})$ was added in seven equal portions. ${ }^{31}$ After heating at $70{ }^{\circ} \mathrm{C}$ for $3 \mathrm{~h}$ and at $90^{\circ} \mathrm{C}$ for $12 \mathrm{~h}$, the mixture was filtered and washed with hot water. The pale pink filtrate was then slowly acidified with $1 \mathrm{M} \mathrm{HCl}$ to obtain a white precipitate. The precipitate 10 was collected by filtration and dried in vacuo $(7 \mathrm{~g}, 36 \%)$. 
Synthesis of 2,2'-bipyridine-5-carboxylic acid chloride (11)

Compound $10(0.7 \mathrm{~g})$ was stirred in $\mathrm{SOCl}_{2}(40 \mathrm{ml})$ and the mixture was heated at $70{ }^{\circ} \mathrm{C}^{31} \mathrm{After} 3 \mathrm{~h}$, the remaining $\mathrm{SOCl}_{2}$ was removed under reduced pressure. The obtained acid chloride $\mathbf{1 1}$ was used for the synthesis of cellulose derivatives without further purification.

\section{Synthesis of cellulose 2,3,6-tris(pyridinecarboxylate)s (1a-d)}

The acid chlorides 2-pyridinecarboxylic acid chloride, 3-pyridinecarboxylic acid chloride, 4-pyridinecarboxylic acid chloride and 6-chloro-3-pyridinecarboxylic acid chloride were prepared from 2-pyridinecarboxylic acid, 3-pyridinecarboxylic acid, 4-pyridinecarboxylic acid and 6-chloro-3-pyridinecarboxylic acid, respectively, through the reaction with oxalyl chloride in toluene at $60^{\circ} \mathrm{C}$. The cellulose derivatives (1a-d) (Figure 1) were synthesized by the reaction of cellulose with an excess amount of the corresponding acid chlorides in an $\mathrm{N}, \mathrm{N}$ dimethylacetamide-LiCl-pyridine-triethylamine mixture at $80^{\circ} \mathrm{C}$ for $24 \mathrm{~h}$. The derivatives were isolated as methanol-insoluble solids and vacuum-dried. Although 1a was insoluble in any solvent, $\mathbf{1 b}-\mathbf{d}$ could be dissolved in organic solvents, such as $\mathrm{N}, \mathrm{N}$-dimethylacetamide, pyridine and dimethyl sulfoxide. The ${ }^{1} \mathrm{H}$ nuclear magnetic resonance (NMR) spectra for $\mathbf{1 b}-\mathbf{d}$ showed that the hydroxy groups of the cellulose were almost quantitatively converted into ester moieties. 1b: ${ }^{1} \mathrm{H}$ NMR (dimethylsulfoxide (DMSO)- $d_{6}$ ): $\delta=3.5-5.8$ (br, glucose protons, $7 \mathrm{H}$ ), $6.8-8.9$ (aromatic, $12 \mathrm{H}$ ). $1 \mathrm{c}$ : ${ }^{1} \mathrm{H}$ NMR (DMSO- $d_{6}$ ): $\delta=3.4-5.8$ (br, glucose protons, $7 \mathrm{H}$ ), 7.2-8.8 (aromatic, $12 \mathrm{H}$ ). 1d: ${ }^{1} \mathrm{H}$ NMR (DMSO- $d_{6}$ ): $\delta=3.3-5.8$ (br, glucose protons, 7H), 6.9-8.8 (aromatic, 9H).

Synthesis of cellulose 2,3,6-tris(2,2'-bipyridine-5-carboxylate) (2) Cellulose $(0.4 \mathrm{~g})$ was dissolved in a $\mathrm{N}, \mathrm{N}$-dimethylacetamide-LiCl-pyridine mixture at $80^{\circ} \mathrm{C}$, and then an excess amount of $11(2.5 \mathrm{~g})$ was added to the solution. The reaction was continued at $80^{\circ} \mathrm{C}$ for $24 \mathrm{~h}$. The cellulose derivative 2 was isolated as a methanol-insoluble fraction and vacuum-dried $(1.5 \mathrm{~g}, 79 \%)$. The ${ }^{1} \mathrm{H}$ NMR spectrum of $\mathbf{2}$ indicated that the degree of substitution of bipyridyl group was $94 \% .{ }^{1} \mathrm{H}$ NMR (pyridine- $d_{5}$ ): $\delta=3.9-6.4$ (br, glucose protons, 7H), 6.9-9.6 (aromatic, 21H).

Synthesis of regioselectively substituted cellulose derivatives (3-6) The regioselectively substituted cellulose derivatives (3-6) having a bipyridyl group (Figure 2) were prepared as described below. ${ }^{32,33}$ First, the cellulose was dissolved in $\mathrm{N}, \mathrm{N}$-dimethylacetamide-LiCl-pyridine mixture at $80^{\circ} \mathrm{C}$, and then 2.0 equivalent of triphenylmethyl chloride, on the basis of a glucose unit, was added and allowed to react with the primary hydroxy group at the 6-position for $24 \mathrm{~h}$. The cellulose derivative protected at the 6-position was isolated as the methanol-insoluble fraction and vacuum-dried. The 6-O-trityl cellulose was reacted with an excess amount of the corresponding acid chlorides or 3,5dimethylphenyl isocyanate in pyridine at $80^{\circ} \mathrm{C}$. The obtained derivatives were suspended in methanol containing $0.1 \mathrm{M}$ hydrochloric acid to deprotect the trityl group at $\sim 50{ }^{\circ} \mathrm{C}$. The deprotected cellulose derivatives were dissolved in pyridine and allowed to react with an excess amount of corresponding acid chlorides or 3,5-dimethylphenyl isocyanate at $80^{\circ} \mathrm{C}$. After $24 \mathrm{~h}$, the regioselectively substituted cellulose derivatives (3-6) were isolated as a methanolinsoluble fraction and vacuum-dried. The obtained derivatives were analyzed by ${ }^{1} \mathrm{H}$ NMR spectroscopy. The cellulose derivatives $\mathbf{3}$ and $\mathbf{4}$ had 87 and $>99 \%$ bipyridyl groups at the 6-position of a glucose unit, respectively. In the derivatives 5 and $\mathbf{6}$, however, the degree of substitution values of bipyridyl groups at the 2- and 3-positions were 33 and $47 \%$, respectively. The degree of substitution values of $\mathbf{5}$ and $\mathbf{6}$ were low, probably because the acid chloride $\mathbf{1 1}$ could not efficiently react with the cellulose derivatives bearing the bulky trityl group. 3: ${ }^{1} \mathrm{H}$ NMR (DMSO- $\left.d_{6}\right)$ : $=1.6-2.3\left(\mathrm{CH}_{3}(12 \mathrm{H})\right.$ ), 3.4-5.4 (br, glucose protons, 7H), 6.0-9.2 (aromatic, NH, 15H). 4: ${ }^{1} \mathrm{H}$ NMR (DMSO- $d_{6}$ ): $\delta=$ 3.4-5.7 (br, glucose protons, 7H), 6.8-9.0 (aromatic, 17H). 5: ${ }^{1} \mathrm{H}$ NMR (DMSO- $\left.d_{6}\right): \delta=1.7-2.5\left(\mathrm{CH}_{3}(6 \mathrm{H})\right), 3.3-5.9$ (br, glucose protons, $\left.7 \mathrm{H}\right), 6.1-$ 10.3 (aromatic, NH, 18H). 6: ${ }^{1} \mathrm{H}$ NMR (DMSO- $d_{6}$ ): $\delta=3.3-5.9$ (br, glucose protons, $7 \mathrm{H}$ ), 6.6-9.0 (aromatic, $19 \mathrm{H}$ ).

\section{Preparation of stationary phases}

The packing materials were prepared according to the previously described method. ${ }^{34}$ The macroporous silica gel obtained from Daiso Chemical was treated with a large excess of (3-aminopropyl)triethoxysilane in toluene in the presence of a catalytic amount of pyridine at $80^{\circ} \mathrm{C}$ for $20 \mathrm{~h}$. The cellulose derivatives bearing pyridyl and bipyridyl residues were dissolved in chloroform, tetrahydrofuran, pyridine or $\mathrm{N}, \mathrm{N}$-dimethylacetamide, and the solutions were coated on the aminopropyl silica gel as uniformly as possible. The solvents were then evaporated under reduced pressure. The remaining polymer solutions were adsorbed onto the silica gel by the same procedure. The total coating amount of the cellulose derivatives to the silica gel was $25 \mathrm{wt} \%$. Because $1 \mathbf{a}$ was insoluble in any solvent, the packing material based on 1a could not be prepared. The obtained packing materials were packed into stainless steel tubes $(25 \times 0.46 \mathrm{~cm}$ ID or $25 \times 0.20 \mathrm{~cm}$ ID) by a conventional high-pressure slurry packing technique using a model CCP-085 Econo packer pump (Chemco, Osaka, Japan). The plate number was $\sim 3000$ for the columns with $25 \times 0.46 \mathrm{~cm}$ ID and $\sim 1500$ for the columns with $25 \times 0.20 \mathrm{~cm}$ ID for benzene using a hexane/2-propanol $(90 / 10, \mathrm{v} / \mathrm{v})$ mixture as the eluent, at flow rates of 0.5 and $0.1 \mathrm{ml} \mathrm{min}^{-1}$, respectively. The non-retained compound 1,3,5-tri-tert-butylbenzene was used to estimate the dead time $\left(t_{0}\right){ }^{35}$

\section{Instruments}

Chromatographic experiments were performed using a JASCO PU-980 Intelligent HPLC pump equipped with ultraviolet (JASCO 970-UV) and polarimetric (JASCO OR-990) detectors (JASCO, Tokyo, Japan) at room temperature or $60^{\circ} \mathrm{C}$. A solution of a racemate $\left(3 \mathrm{mg} \mathrm{ml}^{-1}, 1-10 \mu \mathrm{l}\right)$ was injected into the chromatographic system by a Rheodyne Medel 7125 injector (Rheodyne, Rohnert Park, CA, USA). The ${ }^{1} \mathrm{H}$ NMR $(400 \mathrm{MHz})$ spectra were taken in pyridine- $d_{5}$ and DMSO- $d_{6}$ at $80^{\circ} \mathrm{C}$ using a Varian Gemini-2000 spectrometer (Varian, Palo Alto, CA, USA).

\section{RESULTS AND DISCUSSION}

Enantioseparation on cellulose derivatives bearing pyridyl and bipyridyl groups

Pyridinecarboxylic acid chlorides and 2,2'-bipyridine-5-carboxylic acid chloride were synthesized according to reported procedures ${ }^{27-31}$ and reacted with cellulose to obtain the derivatives 1a-d and 2-6 bearing pyridyl and bipyridyl residues, respectively. The derivatives 1-6 except for 1a were adsorbed onto aminopropyl silica gel, and the obtained packing materials were packed in stainless steel tubes by the slurry method. ${ }^{34}$

The chiral recognition ability on the cellulose derivatives bearing pyridyl and bipyridyl groups was examined for the following 10 racemates: 2-phenylcyclohexanone (12), Tröger base (13), trans-stilbene oxide (14), 1,2,2,2-tetraphenylethanol (15), 2,2'-dihydroxy-6,6'dimethylbiphenyl (16), benzoin (17), flavanone (18), cobalt(III) tris(acetylacetonate) (19), 1-(9-anthryl)-2,2,2-trifluoroethanol (20) and trans-cyclopropanedicarboxylic acid dianilide (21) (Figure 4). Figure 5 shows a chromatogram of the resolution of 16 on the CSP prepared from the cellulose derivative 5 . The enantiomers eluted at the retention times of 23.1 and $33.0 \mathrm{~min}$ showed complete separation. The retention factors, $k_{1}\left(=\left(t_{1}-t_{0}\right) / t_{0}\right)$ and $k_{2}\left(=\left(t_{2}-t_{0}\right) / t_{0}\right)$, were 2.77 and 4.65 , respectively, and the separation factor $\alpha\left(=k_{2} / k_{1}\right)$ was 1.68 .

The results of the resolutions of 12-21 on derivatives $\mathbf{1 b}-\mathbf{d}$ are summarized in Table 1 . The recognition ability of $\mathbf{1 b}$ and $\mathbf{1 c}$ was much lower than that of the commercially available Chiralcel OB, consisting of the cellulose tribenzoate (Figure 1), although the elution orders of the separated enantiomers were the same in these CSPs. Because the pyridyl groups of $\mathbf{1 b}$ and $\mathbf{1} \mathbf{c}$ were located far from a chiral glucose unit, these polar groups themselves may cause a non-enantioselective interaction with the racemates. ${ }^{34}$ This explanation was supported by the notably higher capacity factors of $1 \mathbf{b}$ and $1 \mathrm{c}$ for $\mathbf{1 6}, 20$ and 21 compared with that of Chiralcel $\mathrm{OB}$, indicating that the nitrogen of the pyridyl residues in cellulose derivatives $\mathbf{1 b}$ and $\mathbf{1 c}$ had a hydrogen bonding interaction with the racemates bearing hydroxy and amide groups. On the other hand, the cellulose derivative 1d, which has a 
<smiles>O=C1CCCCC1c1ccccc1</smiles><smiles>Cc1ccc2c(c1)CN1CN2Cc2cc(C)ccc21</smiles>

12<smiles>Pc1ccccc1</smiles>

14<smiles>OC(c1ccccc1)C(c1ccccc1)(c1ccccc1)c1ccccc1</smiles>

15<smiles>Cc1cccc(O)c1Cc1cccc(O)c1C</smiles>

16<smiles>O=C(c1ccccc1)C(O)c1ccccc1</smiles>

17<smiles>O=C1CC(c2ccccc2)Oc2ccccc21</smiles>

18

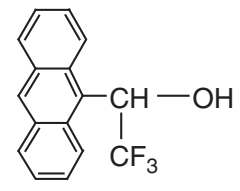

20

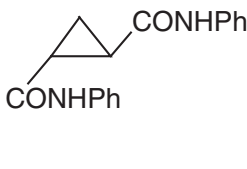

21<smiles>NC(Cc1ccccc1)C(=O)O</smiles>

22<smiles>NC(Cc1cnc[nH]1)C(=O)O</smiles>

23

Figure 4 Structures of racemates 12-23.

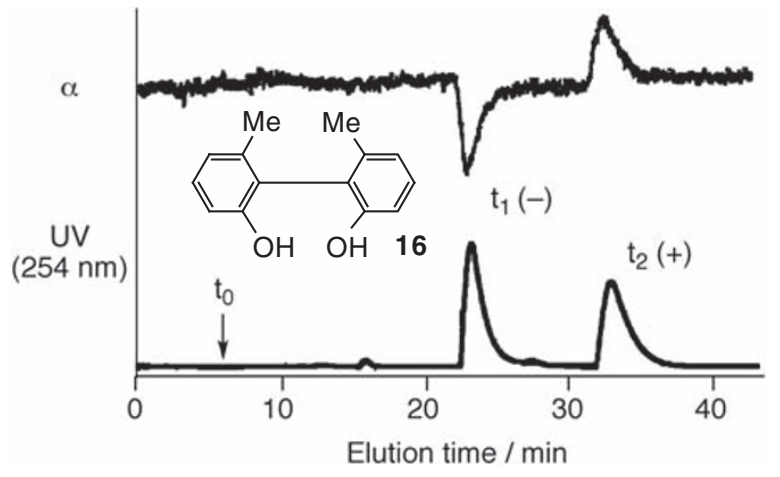

Figure 5 Chiral separation of rac-2,2'-dihydroxy-6,6'-dimethylbiphenyl 16 on cellulose derivative 5 . Column dimensions: $25 \times 0.20 \mathrm{~cm}$ (inner diameter); flow rate: $0.1 \mathrm{ml} \mathrm{min}^{-1}$; eluent: hexane/2-propanol (90:10).

Table 1 Resolutions of $12-21$ on cellulose derivatives bearing pyridyl residues $1 \mathrm{~b}-\mathrm{d}$ and Chiralcel $\mathrm{OB}^{\mathrm{a}}$

\begin{tabular}{|c|c|c|c|c|c|c|c|c|}
\hline \multirow[b]{2}{*}{ Racemates } & \multicolumn{2}{|l|}{$1 b$} & \multicolumn{2}{|l|}{$1 c$} & \multicolumn{2}{|l|}{$1 d$} & \multicolumn{2}{|c|}{ Chiralcel $O B^{b}$} \\
\hline & $k_{1}^{\prime}$ & $\alpha$ & $k_{1}^{\prime}$ & $\alpha$ & $k_{1}^{\prime}$ & $\alpha$ & $k_{1}^{\prime}$ & $\alpha$ \\
\hline 12 & $1.54(-)$ & $\sim 1$ & $1.25(-)$ & 1.11 & $1.82(-)$ & 1.08 & $3.95(-)$ & 1.64 \\
\hline 13 & $0.49(+)$ & $\sim 1$ & $0.27(+)$ & $\sim 1$ & 0.48 & 1.00 & $1.29(-)$ & $\sim 1$ \\
\hline 14 & $0.55(+)$ & $\sim 1$ & $0.50(+)$ & 1.14 & $0.50(+)$ & 1.17 & $0.91(+)$ & 1.44 \\
\hline 15 & 2.36 & 1.00 & $0.57(+)$ & $\sim 1$ & 0.73 & 1.00 & $2.21(+)$ & 1.20 \\
\hline 16 & $11.6(-)$ & 1.09 & $6.82(-)$ & 1.12 & $1.61(-)$ & 1.11 & $1.03(-)$ & 1.30 \\
\hline 17 & $6.03(-)$ & $\sim 1$ & $5.16(+)$ & $\sim 1$ & $4.43(-)$ & 1.05 & $4.16(+)$ & 1.23 \\
\hline 18 & 1.99 & 1.00 & 1.64 & 1.00 & 2.61 & 1.00 & $2.53(-)$ & $\sim 1$ \\
\hline 19 & 0.56 & 1.00 & 0.13 & 1.00 & 0.61 & 1.00 & $1.27(+)$ & $\sim 1$ \\
\hline 20 & 6.63 & 1.00 & 3.25 & 1.00 & 2.03 & 1.00 & 2.14 & 1.00 \\
\hline 21 & 4.07 & 1.00 & $2.80(-)$ & $\sim 1$ & 1.62 & 1.00 & $2.21(+)$ & 1.26 \\
\hline
\end{tabular}

The signs in parentheses represent the optical rotation of the first-eluted enantiomers. aColumn dimensions: $25 \times 0.20 \mathrm{~cm}$ (inner diameter); flow rate: $0.1 \mathrm{ml} \mathrm{min}{ }^{-1}$; eluent: hexane $/ 2$ propanol (90:10)

bColumn dimensions: $25 \times 0.46 \mathrm{~cm}$ (inner diameter); flow rate: $0.5 \mathrm{ml} \mathrm{min}^{-1}$.

chloro group at the para position, exhibited smaller capacity factors and a higher recognition ability than $\mathbf{1 b}$. This suggests that the chloro substituent may inactivate a hydrogen bonding interaction at the nitrogen of the pyridyl residues due to its steric hindrance and electron-withdrawing property.
Table 2 shows the resolution results of 12-21 on the derivatives 2-6. The cellulose derivative $\mathbf{2}$ bearing bipyridyl residues at all 2-, 3- and 6-positions of a glucose ring showed high capacity factors for the racemates, probably due to hydrogen bonding with the bipyridyl groups. However, its recognition ability was very low, and only 12 could be separated with a small $\alpha$-value. The bipyridyl groups may be too far from the main chain to function as effective chiral recognition sites. ${ }^{34}$ On the other hand, the regioselectively substituted derivatives 3-6 exhibited a relatively higher chiral recognition ability than the totally substituted cellulose derivative 2. Compared with a commercially available cellulosebased chiral column, Chiralcel OD, which consists of cellulose tris(3,5-dimethylphenylcarbamate) and is known to be one of the most powerful CSPs (Figure 1), the racemates 12, 13, 16 and 19 and the racemates 12 and $\mathbf{1 6}$ were more efficiently separated on 3 and 5, respectively. Meanwhile, 4 and 6 could separate the racemates 13 and 20 and the racemates 13 and 21, respectively, with larger $\alpha$-values than Chiralcel OB.

Enantioseparation on cellulose derivatives 2-6 having coordinated $\mathrm{Cu}(\mathrm{II})$ ion

The chiral recognition abilities of the derivatives (2-6) were also evaluated after the coordination of $\mathrm{Cu}$ (II) ion (Table 3 ). The chromatographic behavior was significantly altered before and after the coordination of $\mathrm{Cu}$ (II) ion to the bipyridyl residues. Although the recognition ability on these derivatives was decreased for many racemates after coordination, the racemates 14 and 18 were newly resolved on $\mathbf{2}$ and 4, respectively, and some racemates were more efficiently resolved compared with before coordination (Figure 6). In addition, the elution order of the enantiomers of $\mathbf{1 5}$ was reversed on the CSPs prepared from 3. To understand the background of these changes in recognition ability, the structures of $\mathbf{2}$ before (Figure 7a) and after (Figure $7 \mathrm{~b}$ ) the coordination of $\mathrm{Cu}(\mathrm{II})$ ion have been estimated on the basis of the left-handed 3/2-helical structure of cellulose tribenzoate determined by X-ray structural analysis. ${ }^{36}$ Because the higher-ordered structure of $\mathbf{2}$ is not stabilized by an effective intramolecular interaction, such as hydrogen bonds, the orientation of the side chains of $\mathbf{2}$ may be easily changed through the coordination of $\mathrm{Cu}(\mathrm{II})$ ions to the bipyridyl residues (Figure $7 \mathrm{~b}$ ). Moreover, because of the property change due to the coordination itself, this structural difference of the cellulose derivative $\mathbf{2}$ is expected to provide a change in the chiral recognition ability, resulting from the environmental change around the interaction sites, such as ester and carbamate groups. 
Table 2 Resolutions of $12-21$ on cellulose derivatives bearing bipyridyl residue 2-6 and Chiralcel ODa

\begin{tabular}{|c|c|c|c|c|c|c|c|c|c|c|c|}
\hline \multirow[b]{2}{*}{ Racemates } & \multicolumn{2}{|c|}{$2^{\mathrm{b}}$} & \multicolumn{2}{|c|}{3} & \multicolumn{2}{|c|}{4} & \multicolumn{2}{|c|}{5} & \multicolumn{2}{|c|}{6} & \multirow{2}{*}{$\begin{array}{c}\text { Chiralcel } O D^{\mathrm{b}} \\
\alpha\end{array}$} \\
\hline & $k_{1}^{\prime}$ & $\alpha$ & $k_{1}^{\prime}$ & $\alpha$ & $k_{1}^{\prime}$ & $\alpha$ & $k_{1}^{\prime}$ & $\alpha$ & $k_{1}^{\prime}$ & $\alpha$ & \\
\hline 13 & $1.11(+)$ & $\sim 1$ & $0.98(+)$ & 1.70 & $1.21(+)$ & 1.30 & $1.93(+)$ & $\sim 1$ & $1.25(+)$ & 1.29 & $1.26(+)$ \\
\hline 14 & $0.91(+)$ & $\sim 1$ & $0.74(+)$ & 1.24 & $0.94(+)$ & 1.22 & $0.82(+)$ & 1.32 & $1.04(+)$ & 1.15 & $2.11(-)$ \\
\hline 15 & $2.02(-)$ & $\sim 1$ & $1.92(-)$ & 1.20 & $4.17(+)$ & $\sim 1$ & $2.11(+)$ & $\sim 1$ & 3.37 & 1.00 & $1.27(+)$ \\
\hline 18 & 4.23 & 1.00 & $2.24(-)$ & $\sim 1$ & $4.07(-)$ & $\sim 1$ & 2.76 & 1.00 & 4.25 & 1.00 & $1.40(-)$ \\
\hline 19 & 0.71 & 1.00 & $0.93(+)$ & 1.22 & $1.54(+)$ & $\sim 1$ & $1.12(+)$ & $\sim 1$ & 1.00 & 1.00 & $1.13(+)$ \\
\hline 20 & 7.47 & 1.00 & $2.45(-)$ & $\sim 1$ & $2.63(-)$ & 1.08 & $3.44(-)$ & 1.32 & 4.97 & 1.00 & $2.87(-)$ \\
\hline 21 & 2.58 & 1.00 & $1.71(-)$ & 1.28 & 3.63 & 1.00 & 2.81 & 1.00 & $2.56(-)$ & 1.38 & $2.63(+)$ \\
\hline
\end{tabular}

The signs in parentheses represent the optical rotation of the first-eluted enantiomers.

aColumn dimensions: $25 \times 0.20 \mathrm{~cm}$ (inner diameter); flow rate: $0.1 \mathrm{ml} \mathrm{min}^{-1}$; eluent: hexane/2-propanol (90:10).

bColumn dimensions: $25 \times 0.46 \mathrm{~cm}$ (inner diameter); flow rate: $0.5 \mathrm{ml} \mathrm{min}^{-1}$ '.

Table 3 Resolutions of $12-21$ on cellulose derivatives 2-6 having coordinated $\mathrm{Cu}(\mathrm{II})$ ion ${ }^{\mathrm{a}}$

\begin{tabular}{|c|c|c|c|c|c|c|c|c|c|c|}
\hline \multirow[b]{2}{*}{ Racemates } & \multicolumn{2}{|c|}{$2-C u^{b}$} & \multicolumn{2}{|c|}{ 3-Cu } & \multicolumn{2}{|c|}{ 4-Cu } & \multicolumn{2}{|c|}{$5-C u$} & \multicolumn{2}{|c|}{$6-C u$} \\
\hline & $k_{1}^{\prime}$ & $\alpha$ & $k_{1}^{\prime}$ & $\alpha$ & $k_{1}^{\prime}$ & $\alpha$ & $k_{1}^{\prime}$ & $\alpha$ & $k_{1}^{\prime}$ & $\alpha$ \\
\hline 12 & $1.59(-)$ & $\sim 1$ & $0.80(-)$ & 1.28 & $2.18(-)$ & $\sim 1$ & $2.24(-)$ & 1.17 & $2.31(-)$ & $\sim 1$ \\
\hline 13 & 0.53 & 1.00 & $0.56(+)$ & 1.57 & $0.81(+)$ & $\sim 1$ & $1.26(+)$ & $\sim 1$ & $0.84(+)$ & $\sim 1$ \\
\hline 14 & $1.17(+)$ & 1.25 & $0.93(+)$ & 1.15 & $1.29(+)$ & 1.38 & $0.98(+)$ & 1.27 & $1.11(+)$ & $\sim 1$ \\
\hline 15 & 1.56 & 1.00 & $1.00(+)$ & 1.17 & $5.90(+)$ & $\sim 1$ & $2.50(+)$ & 1.00 & 4.33 & 1.00 \\
\hline 16 & 0.66 & 1.00 & $5.72(-)$ & 1.55 & 2.11 & 1.00 & $25.6(-)$ & $\sim 1$ & $2.26(-)$ & $\sim 1$ \\
\hline 17 & 5.68 & 1.00 & $5.22(+)$ & 1.13 & 5.37 & 1.00 & $10.5(+)$ & 1.13 & 5.57 & 1.00 \\
\hline 18 & 2.42 & 1.00 & $2.42(-)$ & $\sim 1$ & $2.61(+)$ & 1.06 & 3.55 & 1.00 & 2.88 & 1.00 \\
\hline 19 & 1.08 & 1.00 & $0.90(+)$ & 1.17 & 2.28 & 1.00 & 1.18 & 1.00 & 1.70 & 1.00 \\
\hline 20 & 2.19 & 1.00 & $7.13(-)$ & 1.15 & 2.28 & 1.00 & 5.46 & 1.00 & 2.45 & 1.00 \\
\hline 21 & 0.22 & 1.00 & 4.82 & 1.00 & 1.88 & 1.00 & 5.21 & 1.00 & 2.28 & 1.00 \\
\hline
\end{tabular}

The signs in parentheses represent the optical rotation of the first-eluted enantiomers.

aColumn dimensions: $25 \times 0.20 \mathrm{~cm}$ (inner diameter); flow rate: $0.1 \mathrm{ml} \mathrm{min}^{-1}$; eluent: hexane/2-propanol (90:10).

bColumn dimensions: $25 \times 0.46 \mathrm{~cm}$ (inner diameter); flow rate: $0.5 \mathrm{ml} \mathrm{min}^{-1}$.
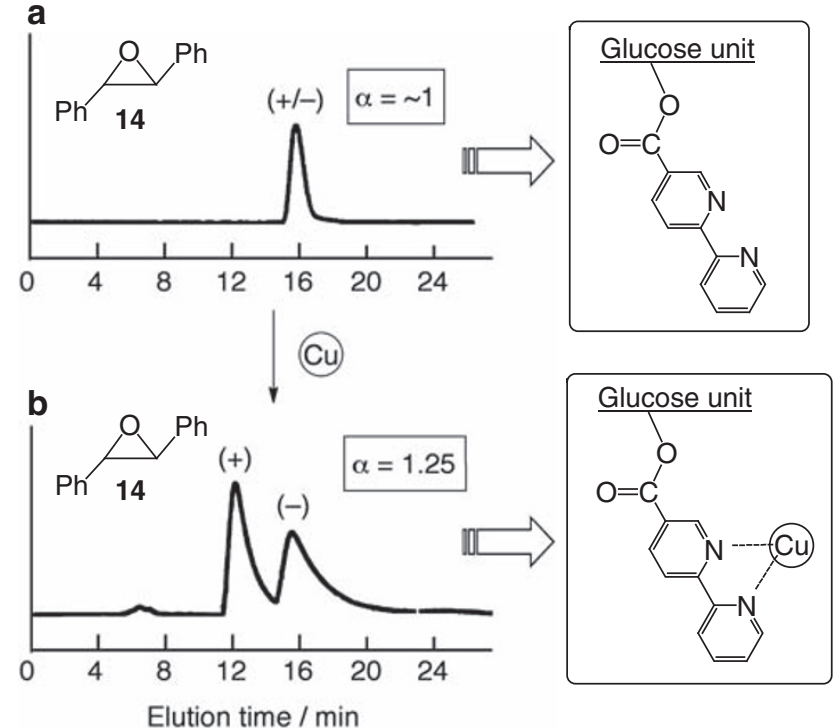

Figure 6 Chromatograms for the resolution of trans-stilbene oxide 14 on 2 with a coordinated $\mathrm{Cu}(\mathrm{II})$ ion.
Chiral ligand-exchange chromatography using cellulose derivatives bearing bipyridyl group

In 1971, Davankov and Rogozhin ${ }^{37}$ developed the first ligandexchange-type CSP, which was prepared by immobilizing $L$-proline onto poly(styrene-co-divinylbenzene) beads. The ligand-exchangetype CSPs were effective for the resolution of chiral compounds bearing polydentate ligands, such as amino acids. ${ }^{38}$ However, the resolution of amino acids was rarely carried out on polysaccharidebased CSPs. Here, the cellulose derivatives bearing bipyridyl residues have been applied for the separation of amino acids using ligandexchange chromatography, because $\mathrm{Cu}$ (II) coordinated in the bipyridyl group is expected to interact with amino acids through the multiple coordination bonds.

Aromatic amino acids, such as phenylalanine (22) and histidine (23), could be separated on the cellulose derivative 6 with $\alpha$-values of 1.12 and 1.37, respectively. Figure 8 shows the resolution of histidine on 6 using an $\mathrm{H}_{2} \mathrm{O} / \mathrm{CH}_{3} \mathrm{OH}$ (90:10) mixture, including $\mathrm{NH}_{4} \mathrm{OAc}$ $(50 \mathrm{~mm})$ and $\mathrm{Cu}(\mathrm{OAc})_{2}(0.5 \mathrm{~mm})$, as an eluent. ${ }^{39,40}$ Although the individual enantiomers showed rather broad overlapping peaks, 6 could partially separate the amino acid. On the other hand, the aliphatic amino acids, such as alanine, leucine, isoleucine, methionine, threonine, valine, proline and serine, could not be separated on these 
a Before coordination of $\mathrm{Cu}(\mathrm{II})$

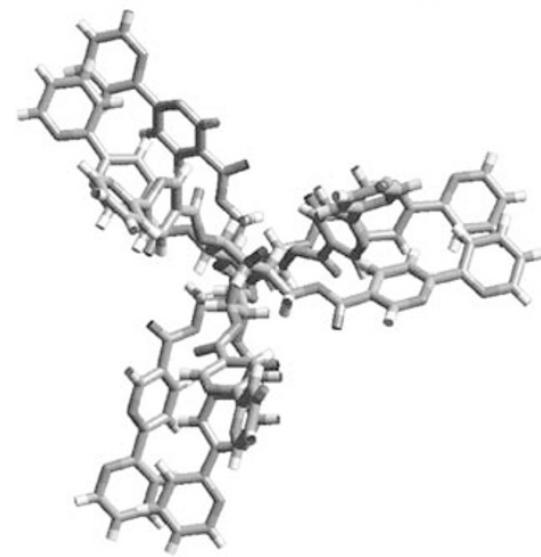

b After coordination of $\mathrm{Cu}$ (II)

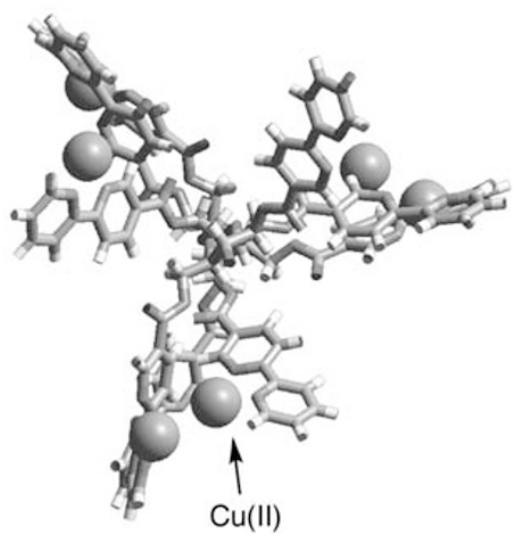

Figure 7 Schematic representation of the structural change of $\mathbf{2}$ before (a) and after (b) coordination of $\mathrm{Cu}(\mathrm{II})$ to the bipyridyl residue. The view is perpendicular to the helix axis, and $\mathbf{2}$ and $\mathrm{Cu}(\mathrm{II})$ are represented by cylinders and van der Waals spheres, respectively.

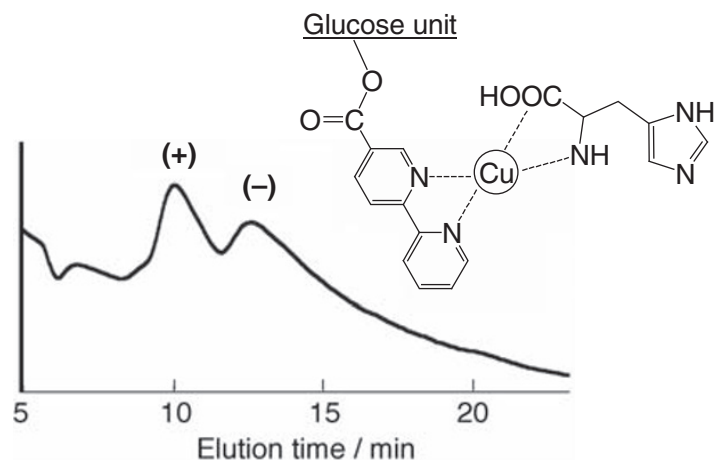

Figure 8 Chiral separation of rac-histidine 23 on 6 . Column dimensions: $25 \times 0.20 \mathrm{~cm}$ (inner diameter); flow rate: $0.1 \mathrm{ml} \mathrm{min}^{-1}$; eluent: $\mathrm{H}_{2} \mathrm{O}-\mathrm{CH}_{3} \mathrm{OH}$ (90:10), $\mathrm{NH}_{4} \mathrm{OAc}(50 \mathrm{~mm}), \mathrm{Cu}(\mathrm{OAc})_{2}(0.5 \mathrm{~mm}) ; \mathrm{pH}: 5.5$; temperature: $60^{\circ} \mathrm{C}$.

derivatives. Therefore, both the coordination bond and the $\pi-\pi$ interaction are expected to have an important role for the separation of amino acids. This result expands the possibility of chiral separation using polysaccharide derivatives. Because the derivatives $\mathbf{2}-\mathbf{5}$ could not separate any amino acids, the environment around the bipyridyl groups is expected to be very important for ligand-exchange chromatography using these derivatives.

\section{CONCLUSIONS}

Cellulose derivatives bearing pyridyl and bipyridyl residues were synthesized, and their chiral recognition abilities as CSPs for HPLC were evaluated. Although the cellulose derivatives bearing pyridyl 1 and bipyridyl 2 residues at all 2-, 3- and 6-positions of a glucose ring showed low chiral recognition, the regioselectively substituted derivatives 3-6 exhibited relatively high chiral recognition. After the coordination of $\mathrm{Cu}$ (II) ion to the bipyridyl residues, their recognition abilities were changed depending on the difference of the higher-order structures of the cellulose derivatives. In addition, the derivatives can be used for ligand-exchange chromatography, and aromatic amino acids, such as phenylalanine and histidine, could be separated on cellulose derivative 6 .

\section{ACKNOWLEDGEMENTS}

This work was partially supported by Daicel Chemical Industries.
1 Rubin, A. E., Tummala, S., Both, D. A., Wang, C. \& Delaney, E. J. Emerging technologies supporting chemical process R\&D and their increasing impact on productivity in the pharmaceutical industry. Chem. Rev. 106, 2794-2810 (2006).

2 Caner, H., Groner, E. \& Levy, L. Trends in the development of chiral drugs. Drug Discov. Today 9, 105-110 (2004).

3 Agranat, I., Caner, H. \& Caldwell, J. Putting chirality to work: The strategy of chiral switches. Nat. Rev. Drug Discov. 1, 753-768 (2002).

4 Maier, N. M., Franco, P. \& Lindner, W. Separation of enantiomers: needs, challenges, perspectives. J. Chromatogr. A 906, 3-33 (2001).

5 Waldeck, B. Biological significance of the enantiomeric purity of drugs. Chirality $\mathbf{5}$, 350-355 (1993)

6 Millership, J. S. \& Fitzpatrick, A. Commonly used chiral drugs: a survey. Chirality $\mathbf{5}$, 573-576 (1993).

7 Caldwell, J. Stereochemical determinants of the nature and consequences of drug metabolism. J. Chromatogr. A 694, 39-48 (1995).

8 Francotte, E. R. \& Lindner, W. (eds). in Chirality in Drug Research (Wiley-VCH, Weinheim, 2006).

9 Toda, F. (ed.). in Enantiomer Separation (Kluwer Academic Publishers, Dordrecht, 2004).

10 Okamoto, Y. \& Ikai, T. Chiral HPLC for efficient resolution of enantiomers. Chem. Soc. Rev. 37, 2593-2608 (2008).

11 Francotte, E. Enantioselective chromatography as a powerful alternative for the preparation of drug enantiomers. J. Chromatogr. A 906, 379-397 (2001).

12 Armstrong, D. W. Optical isomer separation by liquid chromatography. Anal. Chem. 59, 84A-91A (1987).

13 Yamamoto, C. \& Okamoto, Y. Optically active polymers for chiral separation. Bull. Chem. Soc. Jpn. 77, 227-257 (2004).

14 Taylor, D. R. \& Maher, K. Chiral separations by high-performance liquid-chromatography. J. Chromatogr. Sci. 30, 67-85 (1992).

15 Okamoto, Y. Separate optical isomers by chiral HPLC. CHEMTECH 17, 176-181 (1987).

16 Lämmerhofer, M. Chiral recognition by enantioselective liquid chromatography: Mechanisms and modern chiral stationary phases. J. Chromatogr. A 1217, 814-856 (2010).

17 Subramanian, G. (ed.). in Chiral Separation Techniques: A Practical Approach 3rd edn (Wiley-VCH, Weinheim, 2007)

18 Ahuja, S. Chiral Separations by Chromatography (ACS, Washington, DC, 2000).

19 Okamoto, Y. \& Yashima, E. Polysaccharide derivatives for chromatographic separation of enantiomers. Angew. Chem. Int. Ed. 37, 1020-1043 (1998).

20 Yashima, E., Yamamoto, C. \& Okamoto, Y. Polysaccharide-based chiral LC columns. Synlett 344-360 (1998).

21 Stringham, R. W. The use of polysaccharide phases in the separation of enantiomers. Adv. Chromatogr. 44, 257-290 (2006).

22 Tachibana, T. \& Ohnishi, A. Reversed-phase liquid chromatographic separation of enantiomers on polysaccharide type chiral stationary phases. J. Chromatogr. A 906, 127-154 (2001).

23 Ikai, T., Yamamoto, C., Kamigaito, M. \& Okamoto, Y. Immobilized-type chiral packing materials for HPLC based on polysaccharide derivatives. J. Chromatogr. B 875, 2-11 (2008).

24 Yamamoto, C., Yashima, E. \& Okamoto, Y. Structural analysis of amylose tris(3,5dimethylphenylcarbamate) by NMR relevant to its chiral recognition mechanism in HPLC. J. Am. Chem. Soc. 124, 12583-12589 (2002).

$25 \mathrm{lkai}$, T. \& Okamoto, Y. Structure control of polysaccharide derivatives for efficient separation of enantiomers by chromatography. Chem. Rev. 109, 6077-6101 (2009). 
26 Kaida, Y. \& Okamoto, Y. Optical resolution by supercritical fluid chromatography using polysaccharide derivatives as chiral stationary phases. Bull. Chem. Soc. Jpn. 65, 2286-2288 (1992)

27 Wingfield, Jr. H. N. 4-(3-Pyridyl)-4-ketobutyric Acid. J. Org. Chem. 24, 872-873 (1959).

28 Arabaci, G., Yi, T., Fu, H., Porter, M. E., Beebe, K. D. \& Pei, D. $\alpha$-Bromoacetophenone derivatives as neutral protein tyrosine phosphatase inhibitors: Structure-activity relationship. Bioorg. Med. Chem. Lett. 12, 3047-3050 (2002).

29 Rao, P. N. P., Amini, M., Li, H. Y., Habeeb, A. G. \& Knaus, E. E. Design, synthesis, and biological evaluation of 6-substituted-3-(4-methanesulfonylphenyl)-4-phenylpyran-2ones: a novel class of diarylheterocyclic selective cyclooxygenase-2 inhibitors. J. Med. Chem. 46, 4872-4882 (2003).

30 Kröhnke, F. The specific synthesis of pyridines and oligopyridines. Synthesis 1-24 (1976).

31 Fletcher, N. C., Nieuwenhuyzen, M. \& Rainey, S. The isolation and purification of tris2,2'-bipyridine complexes of ruthenium(II) containing unsymmetrical ligands. J. Chem. Soc. Dalton Trans. 2641-2648 (2001).

32 Kaida, Y. \& Okamoto, Y. Optical resolution on regioselectively carbamoylated cellulose and amylose with 3,5-dimethylphenyl and 3,5-dichlorophenyl isocyanates. Bull. Chem. Soc. Jpn. 66, 2225-2232 (1993).
33 Felix, G. Regioselectively modified polysaccharide derivatives as chiral stationary phases in high-performance liquid chromatography. J. Chromatogr. A 906, 171-184 (2001).

34 Okamoto, Y. Kawashima, M. \& Hatada, K. Chromatographic resolution: XI. Controlled chiral recognition of cellulose triphenylcarbamate derivatives supported on silica gel. J. Chromatogr. 363, 173-186 (1986).

35 Koller, H., Rimböck, K. H. \& Mannschreck, A. High-pressure liquid chromatography on triacetylcellulose: characterization of a sorbent for the separation of enantiomers. J. Chromatogr. 282, 89-94 (1983).

36 Steinmeier, H. \& Zugenmaier, P. 'Homogeneous' and 'heterogeneous' cellulose triesters and a cellulose triurethane: synthesis and structural investigations of the crystalline state. Carbohydr. Res. 164, 97-105 (1987).

37 Rogozhin, S. V. \& Davankov, V. A. Ligand chromatography on asymmetric complexforming sorbents as a new method for resolution of racemates. J. Chem. Soc. D: Chem. Commun. 490 (1971).

38 Davankov, V. A. Enantioselective ligand exchange in modern separation techniques. J. Chromatogr. A 1000, 891-915 (2003).

39 Sanaie, N. \& Haynes, C. A. Interpreting the effects of temperature and solvent composition on separation of amino-acid racemates by chiral ligand-exchange chromatography. J. Chromatogr. A 1104, 164-172 (2006).

40 Veigl, E. \& Lindner, W. Epimeric $N$-substituted L-proline derivatives as chiral selectors for ligand-exchange chromatography. J. Chromatogr. A 660, 255-268 (1994). 